\title{
Automatic Continuity of Some Types of Double Derivations on Semisimple Banach Algebras
}

\author{
Amir A. Mohammed and Lamia K. Ismail \\ Department of Mathematics, College of Education \\ of Pure Science University of Mosul, Mosul, Iraq \\ lamiak.ismial88@gmail.com
}

Received

$15 / 01 / 2018$
Accepted

06/03/2018

الخلاصة : (الخ

تبعا لـ بيينا في [9] وعلي ومحمد في [4]، قدمنا المشتقة الثنائية من النمط -c- (h, و

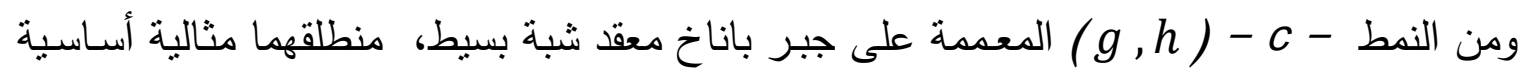

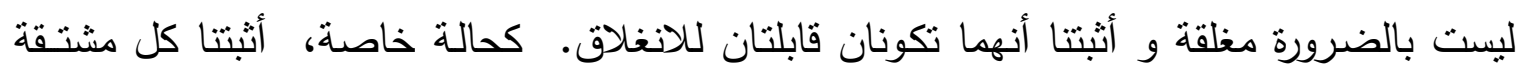

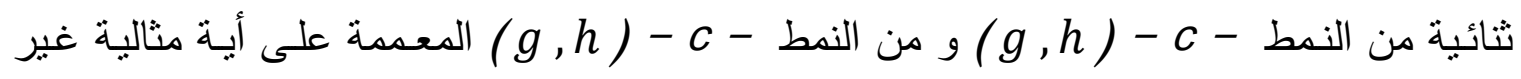
صفرية للجبر - C الأولي تكونان مستمرتان.

\begin{abstract}
Following Villena in [9] and Mohammed and Ali in [4], we introduce partially defined $(g, h)-c$ - double derivation and generalized $(g, h)-c$ double derivation on a semisimple complex Banach algebra whose domain is not necessarily closed, essential ideal and we prove that they are closable. In particular, we show that every $(g, h)$ - $c$-double derivation and generalized $(g, h)-c$ - double derivation defined on any nonzero ideal of a prime $C^{*}$ - algebra are continuous.
\end{abstract}

Keywords: automatic continuity, double derivation, ultraprimness, sliding hump sequence. 


\section{Introduction}

Throughout this paper, $\mathcal{A}$ is a semisimple Banach algebra over complex field and $g, h: \mathcal{A} \rightarrow \mathcal{A}$ are linear mappings. If $g$ and $h$ are the identity maps and if $\mathcal{A}$ with or without identity we may conclude that $g$ and $h$ are continuous by Johnson and Sinclair in [1]. As a consequence, we can assume that $g$ and $h$ are continuous. So, we defined our derivation in this paper as in [5] and [7] as follows: A linear map $D_{1}: \mathcal{A} \rightarrow \mathcal{A}$ is said to be $(g, h)-c$ - double derivation on $\mathcal{A}$ if $D_{1}(a b)=D_{1}(a) b+a D_{1}(b)+g(a) h(b)+h(a) g(b), \forall a, b \in \mathcal{A}$. Similarly, we defined our derivation in this paper as in [8] as follows:

A linear map $D_{2}: \mathcal{A} \rightarrow \mathcal{A}$ is called generalized $(g, h)-c$-double derivation on $\mathcal{A}$ if there exists $(g, h)$ - $c$-double derivation

$D_{1}: \mathcal{A} \rightarrow \mathcal{A}$ such that $D_{2}(a b)=D_{2}(a) b+a D_{1}(b)+g(a) h(b)+$ $h(a) g(b), \forall a, b \in \mathcal{A}$. Recall that, a nonzero ideal $I$ of $\mathcal{A}$ is called essential if for any nonzero ideal $J$ of $\mathcal{A}$ we have $I \cap J \neq\{0\}$. Note that, if $\mathcal{A}$ is prime then any nonzero ideal of $\mathcal{A}$ is essential. By essential defined $(g, h)-c$ - double derivation we mean a linear map $D_{1}: I \rightarrow \mathcal{A}$ such that $I$ is essential and for all $a, b \in I, D_{1}(a b)=D_{1}(a) b+$ $a D_{1}(b)+g(a) h(b)+h(a) g(b)$. Clearly if $g$ or $h$ or both are the zero maps then $D_{1}$ is the usual derivation, so $(g, h)$ - $c$ - double derivation is a generalization of derivation. Similarly, by essential defined generalized $(g, h)-c$ - double derivation we mean a linear map $D_{2}: I \rightarrow \mathcal{A}$ such that $I$ is essential and for all $a, b \in I, D_{2}(a b)=D_{2}(a) b+a D_{1}(b)+g(a)$ $h(b)+h(a) g(b)$.

Clearly if $g$ or $h$ or both are the zero maps and $D_{1}=D_{2}$, then $D_{2}$ is the usual derivation, so generalized $(g, h)-c$ - double derivation is a generalization of derivation. Also if $D_{1}=D_{2}$, then generalized $(g, h)$ - $c$ - double derivation is $(g, h)-c$ - double derivation.

Automatic continuity of derivations are studied by many researcher, we mention some of them of our present work see [1], [2], [5], [6] and [7].

In this paper, we will follow the same lines of [4] and [9]. We will use $D=D_{1}$ or $D_{2}$ when the results are true for both $D_{1}$ and $D_{2}$, otherwise we will use only $D_{1}$ or $D_{2}$.

Let $\mathcal{P}$ denote the set of primitive ideals $P$ of $\mathcal{A}$ such that $I \not \subset P$. The primitive ideal $P$ can be obtained as the kernel of a continuous irreducible representation of $\mathcal{A}$ on a complex Banach

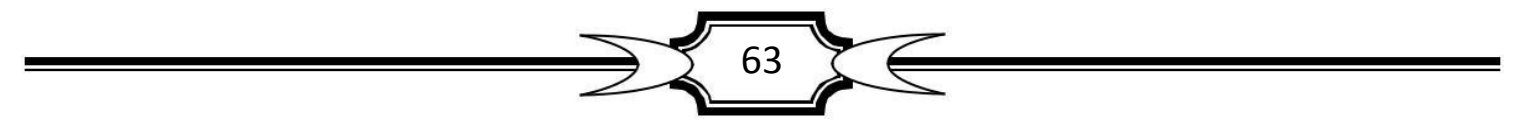




\section{Amir A. Mohammed \& Lamia K. Ismail}

space $X_{P}$, actually the irreducible representation of $\mathcal{A}$ is defined by the following mappings:

$\varphi: \mathcal{A} \rightarrow B L\left(X_{P}\right)$ defined by $\varphi(a)=L_{a}$ and $L_{a}: X_{P} \rightarrow X_{P}$ defined by $L_{a}(x)=a x$ and the $\operatorname{ker}(\varphi)=P$ satisfying $\|a x\| \leq\|a\|\|x\|$, for all $a \in \mathcal{A}, x \in X_{P}$.

Recall that the separating subspace $S(D)$ of $D$ is defined to be the set of those $a$ in $\mathcal{A}$ for which there is a sequence $\left\{a_{n}\right\}$ in $\mathcal{A}$ with $\lim _{n \rightarrow \infty} a_{n}=0$ and $\lim _{n \rightarrow \infty} D\left(a_{n}\right)=a$. It is well known that $D$ is closable if and only if $S(D)=0$, and it is easy to show that $I S(D)+$ $S(D) I \subset S(D)$.

Let $\mathcal{P}_{c}=\{P \in \mathcal{P}: S(D) \subset P\}$ and $\mathcal{P}_{E}=\{P \in \mathcal{P}: S(D) \not \subset P\}$.

Note that $S(D) \subset \cap_{P \in \mathcal{P}_{c}} P=P_{C}$. We will show that $D$ is closed if $P_{C}=0$.

\section{Main Results}

We begin this section by the following fundamental results :

\section{Proposition $1:$ [ 9 ]}

Let $P \in \mathcal{P}$ and $J$ any non necessarily closed ideal of $\mathcal{A}$ satisfying $J \not \subset P$. Then one of the following assertions holds :

1) The ideal of those elements $b \in J$ with $\operatorname{dim} b X_{P}<\infty$ acts irreducibly on $X_{P}$. Accordingly, given $x, y \in X_{P}$ with $x \neq 0$ there is $b \in J$ with $\operatorname{dim} b X_{P}=1$ and $b x=y$.

2) There exist sequences $\left\{b_{n}\right\}$ in $J$ and $\left\{x_{n}\right\}$ in $X_{P}$ satisfying $b_{n} \ldots b_{1} x_{n} \neq 0$ and $b_{n+1} \ldots b_{1} x_{n}=0$ for every $n \in \mathbb{N}$.

Proof : see [ 9 , lemma 1]

Let $\left\{P_{n}\right\}$ be a sequence in $\mathcal{P}$. A sequence $\left\{b_{n}\right\}$ in $I$ is said to be a sliding hump sequence for $\left\{P_{n}\right\}$ if for every $n \in \mathbb{N}$ there exists $x_{n} \in X_{P_{n}}$ such that $b_{n} \ldots b_{1} x_{n} \neq 0$ and $b_{n+1} \ldots b_{1} x_{n}=0$ ( see [9] ).

\section{Proposition 2 :}

If there exists a sliding hump sequence for a sequence $\left\{P_{n}\right\}$ in $\mathcal{P}$, then there is a natural number $n$ for which

i) $S\left(D_{1}\right) \subset P_{n}$. In particular, $S\left(D_{1}\right) \subset P$ if $P_{n}=P$ for every $n \in \mathbb{N}$.

ii) $S\left(D_{2}\right) \subset P_{n}$. In particular, $S\left(D_{2}\right) \subset P$ if $P_{n}=P$ for every $n \in \mathbb{N}$. Proof :

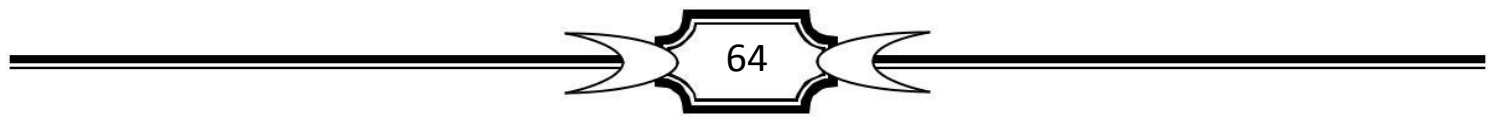


Let $\left\{b_{n}\right\}$ be a sliding hump sequence for $\left\{P_{n}\right\}$ then for every $n \in \mathbb{N}$, there exists $x_{n} \in X_{P_{n}}$ such that $b_{n} \ldots b_{1} x_{n} \neq 0$ and $b_{n+1} \ldots b_{1} x_{n}=0$.

We can certainly assume that $\left\|b_{n}\right\|=\|g\|=\|h\|=\left\|x_{n}\right\|=1$ for every $n \in \mathbb{N}$. We claim that there exist $n \in \mathbb{N}$ and a nonzero $x \in X_{P_{n}}$, such that the map $a \mapsto D(a) x$ from $I$ into $X_{P_{n}}$ is continuous. If the claim fails, then all the maps $a \mapsto D(a) b_{n} \ldots b_{1} x_{n}$ from $I$ into $X_{P_{n}}$ are discontinuous and we can construct inductively a sequence $\left\{a_{n}\right\}$ in $I$ satisfying :

$\left\|D\left(a_{n}\right) b_{n} \ldots b_{1} x_{n}\right\| \geq n+\left\|\sum_{k=1}^{n-1} D\left(a_{k} b_{k} \ldots b_{1}\right) x_{n}\right\|$

$+\left\|D\left(c_{n+1}\right) b_{n+1} \ldots b_{1} x_{n}\right\|$

and $\left\|a_{n}\right\| \leq 2^{-n} \min \left\{\left(1+\left\|D_{1}\left(b_{k} \ldots b_{1}\right)\right\|\right)^{-1}: k=1, \ldots, n\right\}$. Now, we consider the element $c \in \mathcal{A}$ given by $c=\sum_{n=1}^{\infty} a_{n} b_{n} \ldots b_{1}$ and for every $n \in \mathbb{N}$, we write $c_{n}=a_{n}+\sum_{k=n+1}^{\infty} a_{k} b_{k} \ldots b_{n+1}$. Now we will follow the same way of [4] and [9], then we have $c=\sum_{k=1}^{n-1} a_{k} \quad b_{k} \ldots b_{1}+a_{n} b_{n} \ldots b_{1}+c_{n+1} b_{n+1} \ldots b_{1}$. Currently, we will prove the first part of this proposition :

(i) $D_{1}(c)=\sum_{k=1}^{n-1} D_{1}\left(a_{k} b_{k} \ldots b_{1}\right)+D_{1}\left(a_{n}\right) b_{n} \ldots b_{1}+a_{n} D_{1}\left(b_{n} \ldots b_{1}\right)$ $+g\left(a_{n}\right) h\left(b_{n} \ldots b_{1}\right)+h\left(a_{n}\right) g\left(b_{n} \ldots b_{1}\right)+D_{1}\left(c_{n+1}\right) b_{n+1} \ldots b_{1}$ $+c_{n+1} D_{1}\left(\begin{array}{lll}b_{n+1} & \ldots & \left.b_{1}\right)+g\left(c_{n+1}\right) h\left(b_{n+1} \ldots b_{1}\right)\end{array}\right.$ $+h\left(c_{n+1}\right) g\left(b_{n+1} \ldots b_{1}\right)$. Now,

$\left\|D_{1}(c) x_{n}\right\| \geq\left\|D_{1}\left(a_{n}\right) \quad b_{n} \ldots b_{1} x_{n}\right\|-\left\|\sum_{k=1}^{n-1} D_{1}\left(a_{k} b_{k} \ldots b_{1}\right) x_{n}\right\|$ - $\left\|a_{n} D_{1}\left(b_{n} \ldots b_{1}\right) x_{n}\right\|-\left\|g\left(a_{n}\right) h\left(b_{n} \ldots b_{1}\right) x_{n}\right\|$ - $\left\|h\left(a_{n}\right) g\left(b_{n} \ldots b_{1}\right) x_{n}\right\|-\left\|D_{1}\left(c_{n+1}\right) b_{n+1} \ldots b_{1} x_{n}\right\|$ - $\left\|c_{n+1} D_{1}\left(b_{n+1} \ldots b_{1}\right) x_{n}\right\|-\left\|g\left(c_{n+1}\right) h\left(b_{n+1} \ldots b_{1}\right) x_{n}\right\|$ - $\left\|h\left(c_{n+1}\right) g\left(b_{n+1} \ldots b_{1}\right) x_{n}\right\|$, then by $(1)$ we have $\left\|D_{1}(c) x_{n}\right\| \geq n-\left\|a_{n} D_{1}\left(b_{n} \ldots b_{1}\right) x_{n}\right\|-\left\|g\left(a_{n}\right) h\left(b_{n} \ldots b_{1}\right) x_{n}\right\|$ - $\left\|h\left(a_{n}\right) g\left(b_{n} \ldots b_{1}\right) x_{n}\right\|-\left\|c_{n+1} D_{1}\left(b_{n+1} \ldots b_{1}\right) x_{n}\right\|$ - $\left\|g\left(c_{n+1}\right) h\left(b_{n+1} \ldots b_{1}\right) x_{n}\right\|-\left\|h\left(c_{n+1}\right) g\left(b_{n+1} \ldots b_{1}\right) x_{n}\right\| . .(2)$

As a consequence, $\left\|a_{n} D_{1}\left(b_{n} \ldots b_{1}\right) x_{n}\right\| \leq\left\|a_{n}\right\|\left\|D_{1}\left(b_{n} \ldots b_{1}\right)\right\|$ $\leq 1$

Also, $\left\|g\left(a_{n}\right) h\left(b_{n} \ldots b_{1}\right) x_{n}\right\| \leq\|g\|\left\|a_{n}\right\|\|h\|\left\|b_{n}\right\| \ldots\left\|b_{1}\right\|\left\|x_{n}\right\|$ $\leq\left\|a_{n}\right\| \leq 1$

Hence, $\left\|h\left(a_{n}\right) g\left(b_{n} \ldots b_{1}\right) x_{n}\right\| \leq\|h\|\left\|a_{n}\right\|\|g\|\left\|b_{n}\right\| \ldots\left\|b_{1}\right\|\left\|x_{n}\right\|$ $\leq\left\|a_{n}\right\| \leq 1$

Now, we will follow the same way of [4] and [9 ], then we have

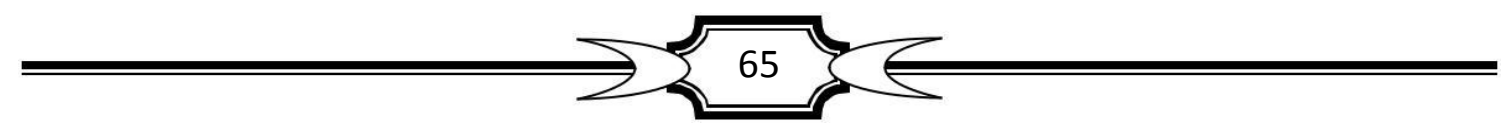




\section{Amir A. Mohammed \& Lamia K. Ismail}

$\left\|c_{n+1}\right\| \leq 2\left\|a_{n+1}\right\|$

So, $\left\|c_{n+1} D_{1}\left(b_{n+1} \ldots b_{1}\right) x_{n}\right\| \leq\left\|c_{n+1}\right\|\left\|D_{1}\left(b_{n+1} \ldots b_{1}\right)\right\|$, then by (6)

$$
\begin{aligned}
& \leq 2\left\|a_{n+1}\right\|\left\|D_{1}\left(b_{n+1} \ldots b_{1}\right)\right\| \\
& \leq 2
\end{aligned}
$$

Also, $\left\|g\left(c_{n+1}\right) h\left(b_{n+1} \ldots b_{1}\right) x_{n}\right\| \leq\|g\|\left\|c_{n+1}\right\|\|h\|\left\|b_{n+1}\right\| \ldots$ $\left\|b_{1}\right\|\left\|x_{n}\right\|, \quad$ then by (6)

$$
\leq 2\left\|a_{n+1}\right\|
$$$$
\leq 2
$$

And, $\left\|h\left(c_{n+1}\right) g\left(b_{n+1} \ldots b_{1}\right) x_{n}\right\| \leq\|h\|\left\|c_{n+1}\right\|\|g\|\left\|b_{n+1}\right\| \ldots$ $\left\|b_{1}\right\|\left\|x_{n}\right\|, \quad$ then by (6)

$$
\begin{aligned}
& \leq 2\left\|a_{n+1}\right\| \\
& \leq 2
\end{aligned}
$$

Then by putting (3), (4), (5), (7), (8) and (9) in (2) we get that $\left\|D_{1}(c) x_{n}\right\| \geq n-9 \quad \forall n \in \mathbb{N}$, then $\left\|D_{1}(c)\right\| \geq\left\|D_{1}(c) x_{n}\right\| \geq n-9$ $\forall n \in \mathbb{N}$. This contradiction proves our claim.

Let $m \in \mathbb{N}$ such that map $a \mapsto D_{1}(a) x$ from $I$ into $X_{P_{m}}$ is continuous for some nonzero $x \in X_{P_{m}}$ and let $X$ be the set of all $x \in X_{P_{m}}$ satisfying this property, $X$ is a nonzero $I$-submodule of $X_{P_{m}}$; therefore, we conclude that $X=X_{P_{m}}$. Let $a \in S\left(D_{1}\right)$ then $\lim _{n \rightarrow \infty} D_{1}\left(a_{n}\right)=a$ for a suitable sequence $\left\{a_{n}\right\}$ in $I$ with $\lim _{n \rightarrow \infty} a_{n}=0$, then $a x=\lim _{n \rightarrow \infty} D_{1}\left(a_{n}\right) x=0$, for every $x \in X_{P_{m}}$ and therefore, $a \in P_{m}$. That means $S\left(D_{1}\right) \subset P_{m}$.

(ii) The proof is similar to the proof of that of first part of this proposition.

\section{Proposition 3 : [9]}

Let $P \in \mathcal{P}$ and $J$ any subspace of $\mathcal{A}$ satisfying $I J+J I \subset J$ and $J \not \subset$ $P$. Then $J x=X_{P}$ for every nonzero $x \in X_{P}$.

Proof : see [ 9 , lemma 3 ]

\section{Proposition 4 :}

Let $P \in \mathcal{P}$ and $J$ any non necessarily closed ideal of $\mathcal{A}$ contained in I. If there is an element $b \in J$ such that $b \notin P$, and $\operatorname{dim} b J b<\infty$. Then $S\left(D_{1}\right) \subset P$ and $S\left(D_{2}\right) \subset P$.

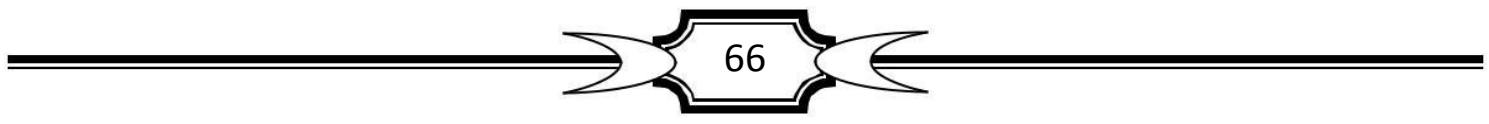


proof :

Note that, since $\operatorname{dim} b J b<\infty$ then the map $a \longmapsto D(b J b)$ is continuous, let $a \in S(D)$, then there exists a sequence $\left\{a_{n}\right\} \subset I$ such that $\lim _{n \rightarrow \infty} a_{n}=0$ and $\lim _{n \rightarrow \infty} D\left(a_{n}\right)=a$. Thus $\lim _{n \rightarrow \infty} b a_{n} b=0$ and $\lim _{n \rightarrow \infty} D\left(b a_{n} b\right)=0$. Since $g$ and $h$ are continuous linear maps, then $\lim _{n \rightarrow \infty} g\left(a_{n}\right)=0$ and $\lim _{n \rightarrow \infty} h\left(a_{n}\right)=0$, also $\lim _{n \rightarrow \infty} b a_{n}=0$ thus $\lim _{n \rightarrow \infty} g\left(b a_{n}\right)=0$ and $\lim _{n \rightarrow \infty} h\left(b a_{n}\right)=0$. Firstly, we will prove $S\left(D_{1}\right) \subset P$. Now, for all $b \in I,\left\{a_{n}\right\} \subset I$, we have:

$$
\begin{aligned}
\lim _{n \rightarrow \infty} D_{1}\left(b a_{n} b\right)= & \lim _{n \rightarrow \infty}\left[D_{1}\left(b a_{n}\right) b+b a_{n} D_{1}(b)+g\left(b a_{n}\right) h(b)+h\left(b a_{n}\right) g(b)\right] \\
= & \lim _{n \rightarrow \infty}\left[D_{1}(b) a_{n} b+b D_{1}\left(a_{n}\right) b+g(b) h\left(a_{n}\right) b+h(\mathrm{~b})\right. \\
& \left.g\left(a_{n}\right) b+b a_{n} D_{1}(b)+g\left(b a_{n}\right) h(b)+h\left(b a_{n}\right) g(b)\right] \\
= & b a b=0 \quad \forall a \in S\left(D_{1}\right) \text { hence } b S\left(D_{1}\right) b=0
\end{aligned}
$$

Since $b \notin P$ then $b X_{P} \neq 0$, if we assume that $S\left(D_{1}\right) \not \subset P$ then by Proposition 3 we have $S\left(D_{1}\right) b X_{P}=X_{P}$ thus $b S\left(D_{1}\right) b X_{P}=b X_{P}=0$ Which gives $b \in P$ this is contradiction; therefore, $S\left(D_{1}\right) \subset P$.

Secondly, we will prove $S\left(D_{2}\right) \subset P$. Since $\lim _{n \rightarrow \infty} D_{2}\left(a_{n}\right)=a$; therefore, $\lim _{n \rightarrow \infty} b D_{2}\left(a_{n}\right)=b a$ this implies that $\lim _{n \rightarrow \infty} D_{2}\left(\begin{array}{ll}b a_{n}\end{array}\right)=b a$, Now, for all $b \in I,\left\{a_{n}\right\} \subset I$,

we have:

$$
\begin{aligned}
\lim _{n \rightarrow \infty} D_{2}\left(b a_{n} b\right)= & \lim _{n \rightarrow \infty}\left[D_{2}\left(b a_{n}\right) b+b a_{n} D_{1}(b)+g\left(b a_{n}\right) h(b)+h\left(b a_{n}\right) g(b)\right] \\
= & \lim _{n \rightarrow \infty} D_{2}\left(b a_{n}\right) b+\lim _{n \rightarrow \infty} b a_{n} D_{1}(b)+\lim _{n \rightarrow \infty} g\left(b a_{n}\right) h(b) \\
& +\lim _{n \rightarrow \infty} h\left(b a_{n}\right) g(b) \\
= & b a b=0 \forall a \in S\left(D_{2}\right) \text { hence } b S\left(D_{2}\right) b=0
\end{aligned}
$$

Since $b \notin P$ then $b X_{P} \neq 0$, if we assume that $S\left(D_{2}\right) \not \subset P$ then by Proposition 3 we have $S\left(D_{2}\right) b X_{P}=X_{P}$ then $b S\left(D_{2}\right) b X_{P}=b X_{P}=0$ that means $b \in P$ this is contradiction; therefore, $S\left(D_{2}\right) \subset P$.

The proof of the following result may be obtained in the same way as in [ 9 , theorem 5 ] applying the above propositions 2 and 4.

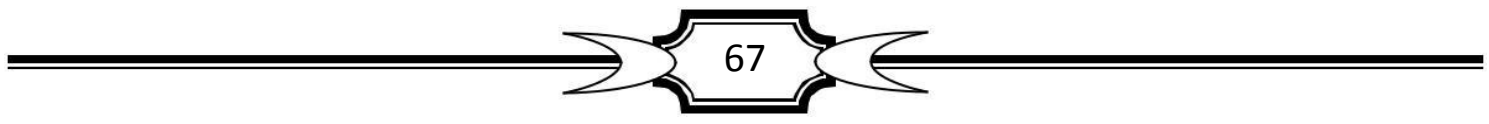




\section{Amir A. Mohammed \& Lamia K. Ismail}

Proposition 5 : $D_{1}$ and $D_{2}$ are closable.

Proof : Obvious.

A Banach algebra $\mathcal{A}$ is said to be ultraprime if there exists a positive constant $K \geq 0$ such that $K\|a\|\|b\| \leq\left\|M_{a, b}\right\| \forall a, b \in$ $\mathcal{A}$, where $M_{a, b}$ is the tow - sided multipliplication operator on $\mathcal{A}$ defined by: $M_{a, b}(x)=\operatorname{axb}($ see [9] $)$.

In [ 3, proposition 2.3 ] it was proved that every prime $C^{*}$ - algebra is an ultraprime Banach algebra, where $K=1$.

\section{Theorem 6 :}

Let $D_{1}$ and $D_{2}$ be closable $(g, h)$-c-double derivation and generalized $(g, h)-c$-double derivation respectively defined on a nonzero ideal I of an ultraprime Banach algebra, then $D_{1}$ and $D_{2}$ are continuous.

proof :

Since $g$ and $h$ are continuous; therefore, there are positive constants $\varepsilon, \delta \geq 0$ such that $\|g(y)\| \leq \varepsilon\|y\|$ and $\|h(z)\| \leq \delta\|z\| \forall y, z \in \mathcal{A}$.

Firstly, we will prove $D_{1}$ is continuous. Fix $a \in I$, with $\|a\|=1$ and consider the following mapping $f_{1}: \mathcal{A} \rightarrow \mathcal{A}$ define by $f_{1}(x)=D_{1}\left(\begin{array}{ll}x & a\end{array}\right)$ $\forall x \in \mathcal{A}$, we will follow the same way of [4] and [9], then we have $f_{1}$ is continuous; therefore, there is a positive constant $t \geq 0$, such that $\left\|f_{1}(x)\right\| \leq t\|x\| \quad \forall x \in \mathcal{A}$. Let $\|x\|=1$ we have $\left\|f_{1}(x)\right\| \leq t$, thus $\left\|f_{1}(x)\right\|=\left\|D_{1}(x a)\right\| \leq t$. Now, for $b \in I, x \in \mathcal{A}$ we have : $D_{1}(b x a)=D_{1}(b) x a+b D_{1}(x a)+g(b) h(x a)+h(b) g(x a)$, then $D_{1}(b) x a=D_{1}(b x a)-b D_{1}(x a)-g(b) h(x a)-h(b) g(x a)$; therefore, $M_{D_{1}(b), a}(x)=D_{1}(b x a)-b D_{1}(x a)-g(b) h(x a)-h(b) g(x a)$, thus $\left\|M_{D_{1}(b), a}(x)\right\| \leq\left\|D_{1}(b x a)\right\|+\left\|b D_{1}(x a)\right\|+\|g(b) h(x a)\|$ $+\|h(b) g(x a)\|$ $\leq t+\|b\| t+\varepsilon\|b\| \delta\|x a\|+\delta\|b\| \varepsilon\|x a\|$ $\leq 4 t \varepsilon \varepsilon \quad \delta\|b\|\|a\|$.

By taking supremum for both sides we have $\left\|M_{D_{1}(b), a}\right\| \leq 4 t \varepsilon \delta\|b\|\|a\|$. Since $\mathcal{A}$ is ultraprime Banach algebra, then there exists a positive constant

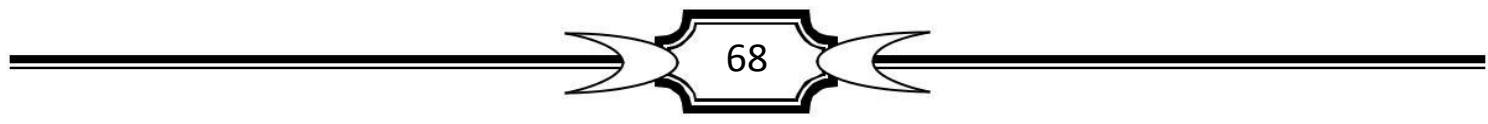


$K \geq 0$ such that $K\|a\|\|b\| \leq\left\|M_{a, b}\right\|$, for all $a, b \in \mathcal{A}$. Then $K \quad\left\|D_{1}(b)\right\|\|a\| \leq\left\|M_{D_{1}(b), a}\right\| \leq 4 t \varepsilon \delta\|b\|\|a\|$, hence $\left\|D_{1}(b)\right\| \leq \frac{4 t \varepsilon \delta}{K}\|b\|, \forall b \in I$. This implies that $D_{1}$ is continuous.

Secondly, we will prove $D_{2}$ is continuous. Fix $a \in I$, with $\|a\|=1$ and consider the following mapping $f_{2}: \mathcal{A} \rightarrow \mathcal{A}$ define by:

$$
f_{2}(x)=D_{2}\left(\begin{array}{ll}
x & a
\end{array}\right) \forall x \in \mathcal{A} \text {, }
$$

we will follow the same way of [4] and [9], then we have $f_{2}$ is continuous; therefore, there is a positive constant $r \geq 0$, such that $\left\|f_{2}(x)\right\| \leq r\|x\| \quad \forall x \in \mathcal{A}$. Let $\|x\|=1$ we have $\left\|f_{2}(x)\right\| \leq r$, thus $\left\|f_{2}(x)\right\|=\left\|D_{2}(x a)\right\| \leq r$. Now, for $b \in I, x \in \mathcal{A}$ we have : $D_{2}(b x a)=D_{2}(b) x a+b D_{1}(x a)+g(b) h(x a)+h(b) g(x a)$, so $D_{2}(b) x a=D_{2}(b x a)-b D_{1}(x a)-g(b) h(x a)-h(b) g(x a)$; therefore, $M_{D_{2}(b), a}(x)=D_{2}(b x a)-b D_{1}(x a)-g(b) h(x a)-h(b) g(x a)$, thus $\left\|M_{D_{2}(b), a}(x)\right\| \leq\left\|D_{2}(b x a)\right\|+\left\|b D_{1}(x a)\right\|+\|g(b) h(x a)\|$ $+\|h(b) g(x a)\|$

$$
\begin{aligned}
& \leq r+\|b\| \frac{4 t \varepsilon \delta}{K}\|x a\|+\varepsilon\|b\| \delta\|x a\|+\delta\|b\| \varepsilon\|x a\| \\
& \leq 7 r t \varepsilon \delta\|b\|\|a\| .
\end{aligned}
$$

By taking supremum for both sides we get $\left\|M_{D_{2}(b), a}\right\| \leq 7 r t \varepsilon \delta\|b\|\|a\|$. Since $\mathcal{A}$ is ultraprime Banach algebra, then there exists a positive constant $m \geq 0$ such that $m\|a\|\|b\| \leq\left\|M_{a, b}\right\|$, for all $a, b \in \mathcal{A}$. Then $m\left\|D_{2}(b)\right\|\|a\| \leq\left\|M_{D_{2}(b), a}\right\| \leq 7 r t \varepsilon \delta\|b\|\|a\|$, hence $\left\|D_{2}(b)\right\| \leq \frac{7 r t \varepsilon \delta}{m}\|b\|, \forall b \in I$. This proves that $D_{2}$ is continuous.

Applying proposition 5 and theorem 6 we can prove the following result :

\section{Corollary 7 :}

Every essentially defined ( $g, h)$ - $c$-double derivation and generalized $(g, h)-c$-double derivation on a nonzero ideal of prime $C^{*}$ - algebra is continuous.

\section{Corollary 8 :}

Every essentially defined derivation on a nonzero ideal of prime $C^{*}$ algebra is continuous.

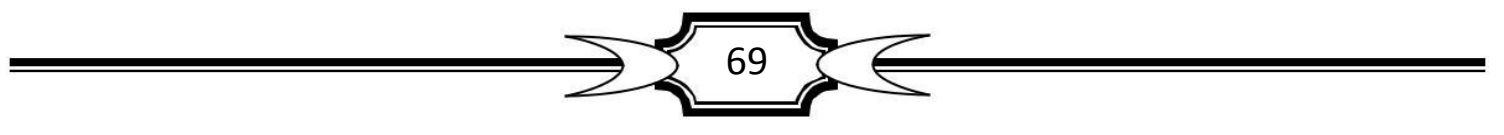


Proof:

i) By corollary 7, taking $g$ or $h$ or both in $D_{1}$ to be the zero maps.

ii) By corollary 7 , let $D_{1}=D_{2}$ and taking $g$ or $h$ or both in $D_{2}$ to be the zero maps.

\section{Remark 9 :}

The above results of this paper are also true for the following derivations:

(1) $D_{3}: I \rightarrow \mathcal{A}$ such that $D_{3}(a b)=D_{3}(a) g(b)+h(a) D_{3}(b)$, for all $a, b \in I$.

(2) $D_{4}: I \rightarrow \mathcal{A}$ such that $D_{4}(a b)=D_{4}(a) g(b)+h(a) D_{3}(b)$, for all $a, b \in I$.

\section{References}

[1] Johnson B. E. and Sinclair A. M., "Continuity of derivations and a problem of Kaplansky" , Amer. J. Math., 90 : 1067 - 1073 (1968) .

[2] Lee $\mathrm{T}-\mathrm{K}$. and Liu $\mathrm{C}-\mathrm{K}$., " Partially defined $\sigma$-derivations on semisimple Banach algebras" , Studia. Math., 190 : 193 - 202 (2009).

[3] Mathieu M., "Elementary operator on prime $C^{*}$ - algebra" , Math. Ann. , $284: 223$ - 244 (1989) .

[4] Mohammed A. A. and Ali S. M. , " On Villena's theorem of automatic continuity of essentially defined derivations on semisimple Banach algebras ", Int. J. of Math. Analysis, 7 : 2931 - 2939 (2013).

[5] Mahdavian Rad H. and Niknam A., "Double derivations, higher double derivations and automatic continuity" , J. of sciences, 24 (2) : 165 - 170 (2013).

[6] Mirzavaziri M. and Moslehian M. S. , "Automatic continuity of $\sigma$-derivations on $C^{*}$-algebras", Proc. Amer. Math. Soc., 134 : 3319 - 3327 (2006) .

[7] Mirzavaziri M. and Omidvar Tehrani E. , “ $(\delta, \epsilon)$ - double derivations on $C^{*}$ - algebras ", Bull. Iranian Math. Soc., 35 : 147 - 154 (2009).

[8] Parky C. and Yun Shinz D., " Generalized $(\theta, \varnothing)$ - derivations on Banach algebras ", Korean J. Math. , 22 (1) : 139 - 150 (2014) .

[9] Villena A. R., " Essentially defined derivations on semisimple Banach algebras", Proc. Edinburgh Math. Soc. , 40 : 175 - 179 (1997). 